\title{
Assessing Software Reliability Using SPC - An Order Statistics Approach
}

\author{
K.Ramchand H Rao Dr. R.Satya Prasad Dr. R.R.L.Kantham \\ ${ }^{1}$ Department of Computer Science, A.S.N. Degree College, Tenali, India \\ ramkolasani@gmail.com \\ ${ }^{2}$ Department of Computer Science, Acharya Nagarjuna University, Guntur, India \\ Profrspegmail.com \\ ${ }^{3}$ Dept. of Statistics, Acharya Nagarjuna University, Guntur, INDIA, \\ kantam_rrlerediffmail.com
}

\begin{abstract}
There are many software reliability models that are based on the times of occurrences of errors in the debugging of software. It is shown that it is possible to do asymptotic likelihood inference for software reliability models based on order statistics or Non-Homogeneous Poisson Processes (NHPP), with asymptotic confidence levels for interval estimates of parameters. In particular, interval estimates from these models are obtained for the conditional failure rate of the software, given the data from the debugging process. The data can be grouped or ungrouped. For someone making a decision about when to market software, the conditional failure rate is an important parameter. Order statistics are used in a wide variety of practical situations. Their use in characterization problems, detection of outliers, linear estimation, study of system reliability, life-testing, survival analysis, data compression and many other fields can be seen from the many books. Statistical Process Control (SPC) can monitor the forecasting of software failure and thereby contribute significantly to the improvement of software reliability. Control charts are widely used for software process control in the software industry. In this paper we proposed a control mechanism based on order statistics of cumulative quantity between observations of time domain failure data using mean value function of Half Logistics Distribution (HLD) based on NHPP.
\end{abstract}

\section{Keywords}

Order Statistics, Statistical Process Control (SPC), Half Logistics Distribution (HLD), NHPP

\section{INTRODUCTION}

The monitoring of Software reliability process is a far from simple activity. In recent years, several authors have recommended the use of SPC for software process monitoring. A few others have highlighted the potential pitfalls in its use[1]. The main thrust of the paper is to formalize and present an array of guidelines in a disciplined process with a view to helping the practitioner in putting SPC to correct use during software process monitoring. Over the years, SPC has come to be widely used among others, in manufacturing industries for the purpose of controlling and improving processes [11]. Our effort is to apply SPC techniques in the software development process so as to improve software reliability and quality [2]. It is reported that SPC can be successfully applied to several processes for software development, including software reliability process. SPC is traditionally so well adopted in manufacturing industry. In general software development activities are more process centric than product centric which makes it difficult to

DOI : $10.5121 /$ ijcsea.2011.1411 
apply SPC in a straight forward manner. The utilization of SPC for software reliability has been the subject of study of several researchers. A few of these studies are based on reliability process improvement models. They turn the search light on SPC as a means of accomplishing high

process maturities. Some of the studies furnish guidelines in the use of SPC by modifying general SPC principles to suit the special requirements of software development [2] (Burr and Owen[3]; Flora and Carleton[4]). It is especially noteworthy that Burr and Owen provide seminal guidelines by delineating the techniques currently in vogue for managing and controlling the reliability of software. Significantly, in doing so, their focus is on control charts as efficient and appropriate SPC tools. It is accepted on all hands that Statistical process control acts as a powerful tool for bringing about improvement of quality as well as productivity of any manufacturing procedure and is particularly relevant to software development also. Viewed in this light, SPC is a method of process management through application of statistical analysis, which involves and includes the defining, measuring, controlling, and improving of the processes [5].

\section{Ordered Statistics}

Order statistics are used in a wide variety of practical situations. Their use in characterization problems, detection of outliers, linear estimation, study of system reliability, life-testing, survival analysis, data compression and many other fields can be seen from the many books [6]. Order statistics deals with properties and applications of ordered random variables and of functions of these variables. The use of order statistics is significant when failures are frequent or inter failure time is less. Let $\mathrm{X}$ denote a continuous random variable with probability density function $\mathrm{f}(\mathrm{x})$ and cumulative distribution function $\mathrm{F}(\mathrm{x})$, and let $\left(\mathrm{X}_{1}, \mathrm{X}_{2}, \ldots, \mathrm{X}_{\mathrm{n}}\right)$ denote a random sample of size $\mathrm{n}$ drawn on $\mathrm{X}$. The original sample observations may be unordered with respect to magnitude. $\mathrm{A}$ transformation is required to produce a corresponding ordered sample. Let $\left(\mathrm{X}_{(1)}, \mathrm{X}_{(2)}, \ldots, \mathrm{X}_{(\mathrm{n})}\right)$ denote the ordered random sample such that $\mathrm{X}_{(1)}<\mathrm{X}_{(2)}<\ldots<\mathrm{X}_{(\mathrm{n})}$; then $\left(\mathrm{X}_{(1)}, \mathrm{X}_{(2)}, \ldots, \mathrm{X}_{(\mathrm{n})}\right)$ are collectively known as the order statistics derived from the parent $\mathrm{X}$. The various distributional characteristics can be known from Balakrishnan and Cohen [7]. The inter-failure time data represent the time lapse between every two consecutive failures. On the other hand if a reasonable waiting time for failures is not a serious problem, we can group the inter-failure time data into non overlapping successive sub groups of size 4 or 5 and add the failure times with in each sub group. For instance if a data of 100 inter-failure times are available we can group them into 20 disjoint subgroups of size 5. The sum total in each subgroup would denote the time lapse between every $5^{\text {th }}$ order statistics in a sample of size 5.In general for inter-failure data of size ' $n$ ', if $r$ (any natural no) less than ' $n$ ' and preferably a factor $n$, we can conviently divide the data into ' $\mathrm{k}$ ' disjoint subgroups $(\mathrm{k}=\mathrm{n} / \mathrm{r})$ and the cumulative total in each subgroup indicate the time between every rth failure. The probability distribution of such a time lapse would be that of the rth ordered statistics in a subgroup of size $r$, which would be equal to rth power of the distribution function of the original variable $(\mathrm{m}(\mathrm{t}))$. The whole process involves the mathematical model of the mean value function and knowledge about its parameters. If the parameters are known they can be taken as they are for the further analysis, if the parameters are not know they have to be estimated using a sample data by any admissible, efficient method of distribution. This is essential because the control limits depend on mean value function, which intern depends on the parameters. If software failures are quite frequent keeping track of inter-failure is tedacious. If failures are more frequent order statistics are preferable.

\section{Model Description}

To calculate the parameter values and control limits using Order Statistics approach, we considered Half Logistic Distribution [8][12]. 
The mean value function of HLD [8] is $m(t)=\frac{a\left(1-e^{-b t}\right)}{\left(1+e^{-b t}\right)}$

To get $\mathrm{m}(\mathrm{t})$ value for $\mathrm{r}^{\text {th }}$ Order Statistics, take $\mathrm{m}(\mathrm{t})$ to the power ' $\mathrm{r}$ '

$$
\begin{aligned}
& {[m(t)]=a^{r}\left(\frac{1-e^{-b t}}{1+e^{-b t}}\right)^{r}} \\
& {\left[m\left(s_{k}\right)\right]=a^{r}\left(\frac{1-e^{-b s_{k}}}{1+e^{-b s_{k}}}\right)^{r}}
\end{aligned}
$$

Derivation with respect to $t$ of equation 4.3.2

$$
\begin{aligned}
& m^{\prime}\left(s_{k}\right)=a^{r} \cdot r\left(\frac{1-e^{-b s_{k}}}{1+e^{-b s_{k}}}\right)^{r-1}\left[\frac{b e^{-b s_{k}}\left(1+e^{-b s_{k}}\right)-\left(1-e^{-b s_{k}}\right)\left(-b e^{-b s_{k}}\right)}{\left(1+e^{-b s_{k}}\right)^{2}}\right] \\
& m^{\prime}\left(s_{k}\right)=\frac{2 b a^{r} \cdot r e^{-b s_{k}}\left(1-e^{-b s_{k}}\right)^{r-1}}{\left(1+e^{-b s_{k}}\right)^{r+1}} \\
& L=e^{-m\left(s_{n}\right)} \prod_{\mathrm{k}=1}^{\mathrm{n}} \mathrm{m}^{\prime}\left(\mathrm{s}_{\mathrm{k}}\right) \\
& \log L=\log \left[e^{-m\left(s_{n}\right)} \prod_{\mathrm{k}=1}^{\mathrm{n}} \mathrm{m}^{\prime}\left(\mathrm{s}_{\mathrm{k}}\right)\right] \\
& =-m\left(s_{n}\right)+\sum_{k=1}^{n} \log \left[\frac{2 b a^{r} \cdot r e^{-b s_{k}}\left(1-e^{-b s_{k}}\right)^{r-1}}{\left(1+e^{-b s_{k}}\right)^{r+1}}\right] \\
& L=-a^{r}\left(\frac{1-e^{-b s_{k}}}{1+e^{-b s_{k}}}\right)^{r}+\sum_{k=1}^{n}\left[\log 2+\log b+r \log a+\log r-b s_{k}+(r-1) \log \left(1-e^{-b s_{k}}\right)-(r+1) \log \left(1+e^{-b s_{k}}\right)\right]
\end{aligned}
$$

Derivation with respect to ' $a$ '

$$
\frac{1}{L} \frac{\partial \mathrm{L}}{\partial \mathrm{a}}=-r a^{r-1}\left(\frac{1-\mathrm{e}^{-\mathrm{bs}_{\mathrm{n}}}}{1+\mathrm{e}^{-\mathrm{bs}_{\mathrm{n}}}}\right)^{\mathrm{r}}+\sum_{k=1}^{n}\left[0+0+\frac{r}{a}+0-0=0-0\right]
$$


International Journal of Computer Science, Engineering and Applications (IJCSEA) Vol.1, No.4, August 2011

$$
=\frac{r}{a}\left(-a^{r}\left(\frac{1-\mathrm{e}^{-\mathrm{bs}_{\mathrm{n}}}}{1+\mathrm{e}^{-\mathrm{bs}_{\mathrm{n}}}}\right)^{r}+n\right)
$$

$\frac{1}{L} \frac{\partial L}{\partial a}=0$

$$
\begin{gathered}
\frac{r}{a}\left[-a^{r}\left(\frac{1-e^{-b s_{n}}}{1+e^{-b s_{n}}}\right)^{r}+n\right]=0 \\
-a^{r}\left(\frac{1-e^{-b s_{n}}}{1+e^{-b s_{n}}}\right)^{r}+n=0 \\
a^{r}=n\left(\frac{1-e^{-b s_{n}}}{1+e^{-b s_{n}}}\right)^{r}
\end{gathered}
$$

$$
\begin{aligned}
\frac{1}{L} \frac{\partial L}{\partial b} & =-a^{r} \cdot r\left(\frac{1-e^{-b s_{n}}}{1+e^{-b s_{n}}}\right)^{r-1}\left[\frac{2 b e^{-b s_{n}}}{\left(1+e^{-b s_{n}}\right)^{2}}\right] \\
\frac{1}{L} \frac{\partial L}{\partial b} & =-a^{r} \cdot r\left(\frac{1-e^{-b s_{n}}}{1+e^{-b s_{n}}}\right)^{r-1}\left[\frac{2 b e^{-b s_{n}}}{\left(1+e^{-b s_{n}}\right)^{2}}\right]+\sum_{k=1}^{n} 0+\frac{1}{b}+0+0-s k+\frac{r-1}{1-e^{-b s_{k}}}\left(b e^{-b s_{k}}\right)-\frac{r+1}{1+e^{-b s_{k}}}\left(-b e^{-b s_{k}}\right) \\
& =\frac{-2 b r a^{r} e^{-b s_{n}}\left(1-e^{-b s_{n}}\right)^{r-1}}{\left(1+e^{-b s_{n}}\right)^{r+1}}+\sum_{k=1}^{n} \frac{1}{b}-s_{k}+b e^{-b s_{k}}\left(\frac{r-1}{1-e^{-b s_{k}}}+\frac{r+1}{1+e^{-b s_{k}}}\right)
\end{aligned}
$$

$$
\frac{1}{L} \frac{\partial L}{\partial b}=\frac{-2 b r a^{r} e^{-b s_{n}}\left(1-e^{-b s_{n}}\right)^{r-1}}{\left(1+e^{-b s_{n}}\right)^{r+1}}+\frac{n}{b}-n s_{k}+\sum_{k=1}^{n} b e^{-b s_{k}}\left[\frac{r-1+(r-1) e^{-b s_{k}}+r+1-(r+1)\left(e^{-b s_{k}}\right)}{1-e^{-2 b s_{k}}}\right]
$$

$$
\frac{1}{L} \frac{\partial L}{\partial b}=\frac{-2 b r a^{r} e^{-b s_{n}}\left(1-e^{-b s_{n}}\right)^{r-1}}{\left(1+e^{-b s_{n}}\right)^{r+1}}+\frac{n}{b}-n s_{k}+\sum_{k=1}^{n} b e^{-b s_{k}}\left[\frac{2 r-2 e^{-b s_{k}}}{1-e^{-2 b s_{k}}}\right]
$$


International Journal of Computer Science, Engineering and Applications (IJCSEA) Vol.1, No.4, August 2011

$$
\begin{aligned}
& g(b)=\frac{-2 b r a^{r} e^{-b s_{n}}\left(1-e^{-b s_{n}}\right)^{r-1}}{\left(1+e^{-b s_{n}}\right)^{r+2}}+\frac{n}{b}-n s_{k}+\sum_{k=1}^{n}\left[\frac{2 b e^{-b s_{k}} r-e^{-b s_{k}}}{1-e^{-2 b s_{k}}}\right] \\
& g(b)=\frac{2 b r e^{-b s_{n}}\left(1-e^{-b s_{n}}\right)^{r-1}}{\left(1+e^{-b s_{n}}\right)^{r+1}} n\left(\frac{1+e^{-b s_{n}}}{1-e^{-b s_{n}}}\right)^{r}+\frac{n}{b}-n s_{k}+\sum_{k=1}^{n}\left[\frac{2 b e^{-b s_{k}} r-e^{-b s_{k}}}{1-e^{-2 b s_{k}}}\right] \\
& =\frac{2 n b e^{-b s_{n}}}{\left(1+e^{-b s_{n}}\right)\left(1-e^{-b s_{n}}\right)}+\frac{n}{b}-n s_{k}+\sum_{k=1}^{n}\left[\frac{2 b e^{-b s_{k}} r-e^{-b s_{k}}}{1-e^{-2 b s_{k}}}\right] \\
& =\frac{2 n b e^{-b s_{n}}}{1-e^{-2 b s_{n}}}+\frac{n}{b}-n s_{k}+\sum_{k=1}^{n}\left[\frac{2 b e^{-b s_{k}} r-e^{-b s_{k}}}{1-e^{-2 b s_{k}}}\right]+2 \sum_{k=1}^{n} \frac{\left(b r e^{-b s_{k}}-b e^{-2 b s_{k}}\right)}{1-e^{-2 b s_{k}}}+2 r \sum_{k=1}^{n} \frac{b e^{-b s_{k}}}{1-e^{-2 b s_{k}}}-2 \sum_{k=1}^{n} \frac{b e^{-2 b s_{k}}}{1-e^{-2 b s_{k}}} \\
& g(b)=\frac{2 n b e^{-b s_{n}}}{1-e^{-2 b s_{n}}}+\frac{n}{b}-n s_{k}+2 r \sum_{k=1}^{n} \frac{b}{e^{b s_{k}}-e^{-b s_{k}}}-2 \sum_{k=1}^{n} \frac{b}{e^{-2 b s_{k}}-1} \\
& g^{\prime}(b)=2 n\left[\frac{\left(e^{-b s_{n}}-b s_{n} e^{b s_{n}}\right)\left(1-e^{-2 b s_{n}}\right)-b e^{-b s_{n}}\left(2 b e^{-b s_{n}}\right)}{\left(1-e^{-2 b s_{n}}\right)^{2}}\right]+n\left(\frac{-1}{b^{2}}\right)-0+2 r \sum_{k=1}^{n} \frac{1 \cdot\left(e^{b s_{k}}-e^{-b s_{k}}\right)-b\left(s_{k} e^{b s_{k}}+s_{k} e^{-b s_{k}}\right)}{\left(e^{b s_{k}}-e^{-b s_{k}}\right)^{2}} \\
& -2 n \sum_{k=1}^{n} \frac{1 \cdot\left(e^{2 b s_{k}}-1\right)-b 2 s_{k} e^{2 b s_{k}}}{\left(e^{2 b s_{k}}-1\right)^{2}} \\
& =2 n \frac{e^{-b s_{n}}\left[\left(1-b s_{n}\right)\left(1-e^{-2 b s_{n}}\right)-2 b^{2} e^{-2 b s_{n}}\right]}{\left(1-e^{-2 b s_{n}}\right)^{2}}+\frac{n}{b^{2}}+2 r \sum_{k=1}^{n} \frac{e^{b s_{k}}-e^{-b s_{k}}-b s_{k}\left(e^{b s_{k}}+e^{-b s_{k}}\right)}{\left(e^{b s_{k}}-e^{-b s_{k}}\right)^{2}} \\
& -2 n \sum_{k=1}^{n} \frac{e^{2 b s_{k}}-1-2 b s_{k} e^{2 b s_{k}}}{\left(e^{2 b s_{k}}-1\right)^{2}}
\end{aligned}
$$

125 


\section{Monitoring the time between failures using control -chart}

The selection of proper SPC charts is essential to effective statistical process control implementation and use. There are many charts which use statistical techniques. It is important to use the best chart for the given data, situation and need [9].There are advances charts that provide more effective statistical analysis. The basic types of advanced charts, depending on the type of data are the variable and attribute charts. Variable control chats are designed to control product or process parameters which are measured on a continuous measurement scale. X-bar, $\mathrm{R}$ charts are variable control charts. Attributes are characteristics of a process which are stated in terms of good are bad, accept or reject, etc. Attribute charts are not sensitive to variation in the process as variables charts. However, when dealing with attributes and used properly, especially by incorporating a real time pareto analysis, they can be effective improvement tools. For attribute data there are : p-charts, c-charts, np-charts, and u-charts. We have named the control chart as Failures Control Chart in this paper. The said control chart helps to assess the software failure phenomena on the basis of the given inter- failure time data

\section{Estimation of Parameters and Control Limits}

Given the data observations and sample size and using equations (3.1),(3.8),(3.9), the parameters

' $a$ ' and 'b' are computed by using the popular NR method. A program written in C was used for this purpose. The equation for mean value function of Half Logistic Distribution is given by

$$
m(t)=a\left[\frac{1-e^{-b t}}{1+e^{-b t}}\right]
$$

The Control limits are obtained as follows: Delete the term 'a' from the mean value function. Equate the remaining function successively to $0.99865,0.00135,0.5$ and solve for ' $t$ ', for half logistic distribution, in order to get the usual Six sigma corresponding control limits, central line.

$$
\begin{aligned}
& \mathrm{F}(\mathrm{t})=\frac{1-e^{-b t}}{1+e^{-b t}}=0.99865 \\
& \Rightarrow 1-e^{-b t}=0.99865\left(1+e^{-b t}\right) \\
& \Rightarrow 1-e^{-b t}=0.99865+0.99865 e^{-b t} \\
& \Rightarrow 1-0.99865=e^{-b t}+0.99865 . e^{-b t} \\
& \Rightarrow 0.00135=(1+0.99865) e^{-b t} \\
& \Rightarrow e^{-b t}=\frac{0.00135}{1.99865}=0.000675456 \\
& \Rightarrow+b t=\log (0.000675456)=+7.300122639 \\
& \text { It gives } \\
& t=\frac{7.300122639}{b}=t_{U} \\
& t=\frac{0.002700002}{b}=t_{L} \\
& t=\frac{1.098612289}{b}=t_{C}
\end{aligned}
$$


The control limits are such that the point above the $\mathrm{m}\left(\mathrm{t}_{\mathrm{U}}\right)(4.1)(\mathrm{UCL})$ is an alarm signal. A point below the $\mathrm{m}\left(\mathrm{t}_{\mathrm{L}}\right)(4.3)$ (LCL) is an indication of better quality of software. A point within the control limits indicates stable process.

\subsection{Developing Failures Chart:}

Given the $n$ inter-failure data the values of $m(t)$ at $T_{c}, T_{u}, T_{L}$ and at the given $n$ inter-failure times are calculated. Then successive differences of the $m(t)$ 's are taken, which leads to $n-1$ values. The graph with the said inter-failure times 1 to $n-1$ on X-axis, the n-1 values of successive differences $m(t)$ 's on $Y$-axis, and the 3 control lines parallel to $X$-axis at $m\left(T_{L}\right), m\left(T_{U}\right), m\left(T_{C}\right)$ respectively constitutes failures control chart to assess the software failure phenomena on the basis of the given inter-failures time data.

\section{Illustration}

The procedure of a failures control chart for failure software process is illustrated with an example here. Table 1 show the time between failures of software product [10].

Table:1 Software failure data reported by Musa(1975) [10]

\begin{tabular}{|c|c|c|c|c|c|c|c|}
\hline Fault & Time & Fault & Time & Fault & Time & Fault & Time \\
\hline 1 & 3 & 35 & 227 & 69 & 529 & 103 & 108 \\
\hline 2 & 30 & 36 & 65 & 70 & 379 & 104 & 0 \\
\hline 3 & 113 & 37 & 176 & 71 & 44 & 105 & 3110 \\
\hline 4 & 81 & 38 & 58 & 72 & 129 & 106 & 1247 \\
\hline 5 & 115 & 39 & 457 & 73 & 810 & 107 & 943 \\
\hline 6 & 9 & 40 & 300 & 74 & 290 & 108 & 700 \\
\hline 7 & 2 & 41 & 97 & 75 & 300 & 109 & 875 \\
\hline 8 & 91 & 42 & 263 & 76 & 529 & 110 & 245 \\
\hline 9 & 112 & 43 & 452 & 77 & 281 & 111 & 729 \\
\hline 10 & 15 & 44 & 255 & 78 & 160 & 112 & 1897 \\
\hline 11 & 138 & 45 & 197 & 79 & 828 & 113 & 447 \\
\hline 12 & 50 & 46 & 193 & 80 & 1011 & 114 & 386 \\
\hline 13 & 77 & 47 & 6 & 81 & 445 & 115 & 446 \\
\hline 14 & 24 & 48 & 79 & 82 & 296 & 116 & 122 \\
\hline 15 & 108 & 49 & 816 & 83 & 1755 & 117 & 990 \\
\hline 16 & 88 & 50 & 1351 & 84 & 1064 & 118 & 948 \\
\hline 17 & 670 & 51 & 148 & 85 & 1783 & 119 & 1082 \\
\hline 18 & 120 & 52 & 21 & 86 & 860 & 120 & 22 \\
\hline 19 & 26 & 53 & 233 & 87 & 983 & 121 & 75 \\
\hline 20 & 114 & 54 & 134 & 88 & 707 & 122 & 482 \\
\hline 21 & 325 & 55 & 357 & 89 & 33 & 123 & 5509 \\
\hline 22 & 55 & 56 & 193 & 90 & 868 & 124 & 100 \\
\hline 23 & 242 & 57 & 236 & 91 & 724 & 125 & 10 \\
\hline 24 & 68 & 58 & 31 & 92 & 2323 & 126 & 1071 \\
\hline 25 & 422 & 59 & 369 & 93 & 2930 & 127 & 371 \\
\hline 26 & 180 & 60 & 748 & 94 & 1461 & 128 & 790 \\
\hline 27 & 10 & 61 & 0 & 95 & 843 & 129 & 6150 \\
\hline 28 & 1146 & 62 & 232 & 96 & 12 & 130 & 3321 \\
\hline 29 & 600 & 63 & 330 & 97 & 261 & 131 & 1045 \\
\hline 30 & 15 & 64 & 365 & 98 & 1800 & 132 & 648 \\
\hline 31 & 36 & 65 & 1222 & 99 & 865 & 133 & 5485 \\
\hline 32 & 4 & 66 & 543 & 100 & 1435 & 134 & 1160 \\
\hline 33 & 0 & 67 & 10 & 101 & 30 & 135 & 1864 \\
\hline 34 & 8 & 68 & 16 & 102 & 143 & 136 & 4116 \\
\hline
\end{tabular}


International Journal of Computer Science, Engineering and Applications (IJCSEA) Vol.1, No.4, August 2011

Table: 2 Parameter estimates and their control limits of 4 and 5 order

\begin{tabular}{|c|c|c|c|c|c|c|}
\hline Data Set & Order & $\mathbf{a}$ & $\mathbf{b}$ & $m\left(t_{U}\right)$ & $m\left(t_{C}\right)$ & $m\left(t_{L}\right)$ \\
\hline \multirow{2}{*}{ Table 1 } & $\mathbf{4}$ & 2.414736 & 0.000727 & 2.411476 & 1.207368 & 0.003260 \\
\cline { 2 - 7 } & $\mathbf{5}$ & 1.933309 & 0.000114 & 1.930699 & 0.966655 & 0.002610 \\
\hline
\end{tabular}

Table: 3 Successive differences of 4 order $\mathbf{m}(t)$ 's of Table 1

\begin{tabular}{|c|c|c|c|c|c|c|c|}
\hline Fault & $\begin{array}{l}\text { 4-order } \\
\text { Cumul- } \\
\text { atives }\end{array}$ & $\mathbf{m}(\mathbf{t})$ & $\begin{array}{c}\text { Successive } \\
\text { Difference's } \\
\text { Of m(t)'s }\end{array}$ & Fault & $\begin{array}{l}\text { 4-order } \\
\text { Cumul- } \\
\text { atives }\end{array}$ & $\mathbf{m}(\mathbf{t})$ & $\begin{array}{c}\text { Successive } \\
\text { Difference's } \\
\text { Of m(t)'s }\end{array}$ \\
\hline 1 & 227 & 0.198799753 & 0.187575193 & 18 & 16358 & 2.414702952 & $2.49182 \mathrm{E}-05$ \\
\hline 2 & 444 & 0.386374945 & 0.263437663 & 19 & 18287 & 2.41472787 & $6.58063 \mathrm{E}-06$ \\
\hline 3 & 759 & 0.649812608 & 0.234105106 & 20 & 20567 & 2.41473445 & 1.43319E-06 \\
\hline 4 & 1056 & 0.883917714 & 0.608610384 & 21 & 24127 & 2.414735884 & $1.11484 \mathrm{E}-07$ \\
\hline 5 & 1986 & 1.492528098 & 0.318281856 & 22 & 28460 & 2.414735995 & 4.70782E-09 \\
\hline 6 & 2676 & 1.810809953 & 0.419004464 & 23 & 32408 & 2.414736 & $2.76672 \mathrm{E}-10$ \\
\hline 7 & 4434 & 2.229814417 & 0.068367032 & 24 & 37654 & 2.414736 & $5.98011 \mathrm{E}-12$ \\
\hline 8 & 5089 & 2.298181449 & 0.022394142 & 25 & 42015 & 2.414736 & $4.84057 \mathrm{E}-14$ \\
\hline 9 & 5389 & 2.320575591 & 0.047885291 & 26 & 42296 & 2.414736 & $2.10498 \mathrm{E}-13$ \\
\hline 10 & 6380 & 2.368460882 & 0.02486038 & 27 & 48296 & 2.414736 & 0 \\
\hline 11 & 7447 & 2.393321262 & 0.006233656 & 28 & 52042 & 2.414736 & 0 \\
\hline 12 & 7922 & 2.399554918 & 0.012395799 & 29 & 53443 & 2.414736 & 0 \\
\hline 13 & 10258 & 2.411950717 & 0.001354859 & 30 & 56485 & 2.414736 & 0 \\
\hline 14 & 11175 & 2.413305576 & 0.000907338 & 31 & 62651 & 2.414736 & 0 \\
\hline 15 & 12559 & 2.414212914 & 0.000256454 & 32 & 64893 & 2.414736 & 0 \\
\hline 16 & 13486 & 2.414469368 & 0.000194113 & 33 & 76057 & 2.414736 & 0 \\
\hline 17 & 15277 & 2.414663481 & $3.94709 \mathrm{E}-05$ & 34 & 76057 & 2.414736 & \\
\hline
\end{tabular}




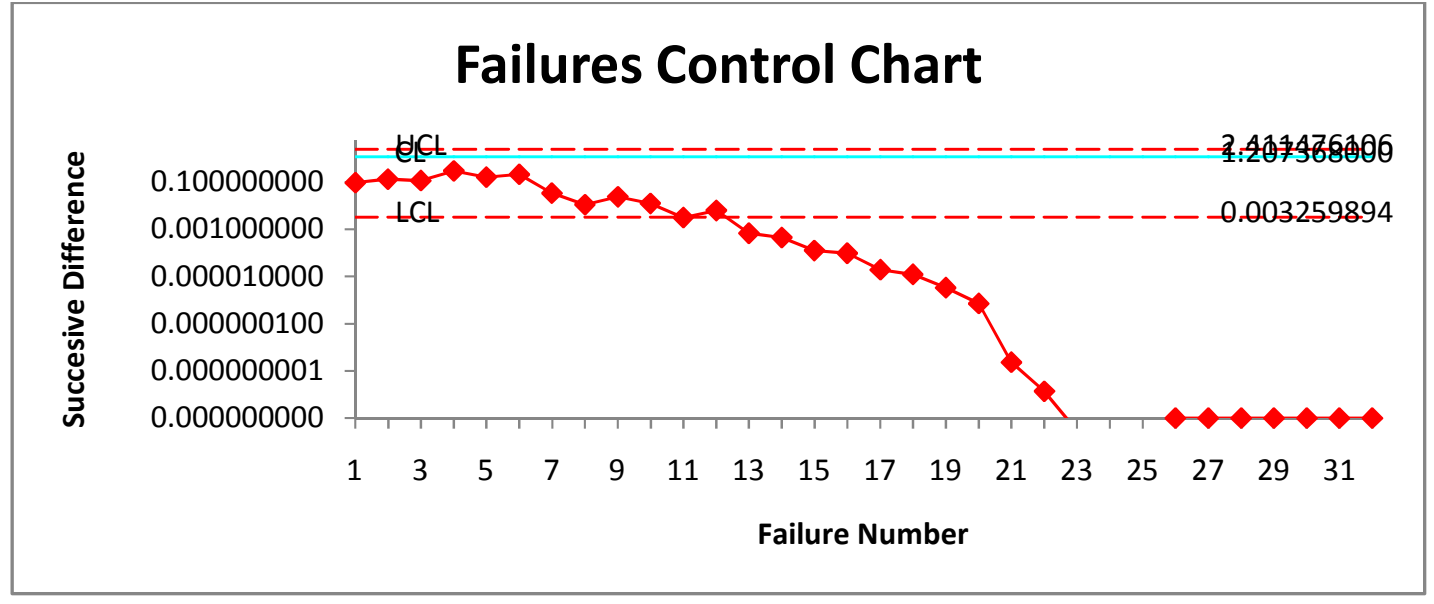

Fig 1: Failures Control Chart of Table 3

Table: 4 Successive differences of 5 order $\mathrm{m}(\mathrm{t})$ 's of Table 1

\begin{tabular}{|c|c|c|c|c|c|c|c|}
\hline Fault & $\begin{array}{l}\text { 5-order } \\
\text { Cumul- } \\
\text { atives }\end{array}$ & $\mathbf{m}(\mathbf{t})$ & $\begin{array}{c}\text { Successive } \\
\text { Difference's } \\
\text { Of m(t)'s }\end{array}$ & Fault & $\begin{array}{l}\text { 5-order } \\
\text { Cumula- } \\
\text { tives }\end{array}$ & $\mathbf{m}(\mathbf{t})$ & $\begin{array}{c}\text { Successive } \\
\text { Difference's } \\
\text { Of m(t)'s }\end{array}$ \\
\hline 1 & 342 & 0.037683152 & 0.025218047 & 15 & 17758 & 1.482215615 & 0.112791696 \\
\hline 2 & 571 & 0.062901199 & 0.043662939 & 16 & 20567 & 1.595007310 & 0.146671923 \\
\hline 3 & 968 & 0.106564138 & 0.111360223 & 17 & 25910 & 1.741679234 & 0.060209283 \\
\hline 4 & 1986 & 0.217924362 & 0.119966118 & 18 & 29361 & 1.801888517 & 0.079206429 \\
\hline 5 & 3098 & 0.337890480 & 0.203633678 & 19 & 37642 & 1.881094946 & 0.020328756 \\
\hline 6 & 5049 & 0.541524158 & 0.027802654 & 20 & 42015 & 1.901423702 & 0.010165625 \\
\hline 7 & 5324 & 0.569326811 & 0.104303350 & 21 & 45406 & 1.911589327 & 0.007940730 \\
\hline 8 & 6380 & 0.673630162 & 0.119182170 & 22 & 49416 & 1.919530057 & 0.004939245 \\
\hline 9 & 7644 & 0.792812332 & 0.210720360 & 23 & 53321 & 1.924469302 & 0.002672472 \\
\hline 10 & 10089 & 1.003532692 & 0.069983093 & 24 & 56485 & 1.927141773 & 0.003114633 \\
\hline 11 & 10982 & 1.073515785 & 0.114193668 & 25 & 62661 & 1.930256406 & 0.002248122 \\
\hline 12 & 12559 & 1.187709454 & 0.136486011 & 26 & 74364 & 1.932504528 & 0.000553008 \\
\hline 13 & 14708 & 1.324195465 & 0.081511891 & 27 & 84566 & 1.933057536 & \\
\hline 14 & 16185 & 1.405707355 & 0.076508259 & & & & \\
\hline
\end{tabular}




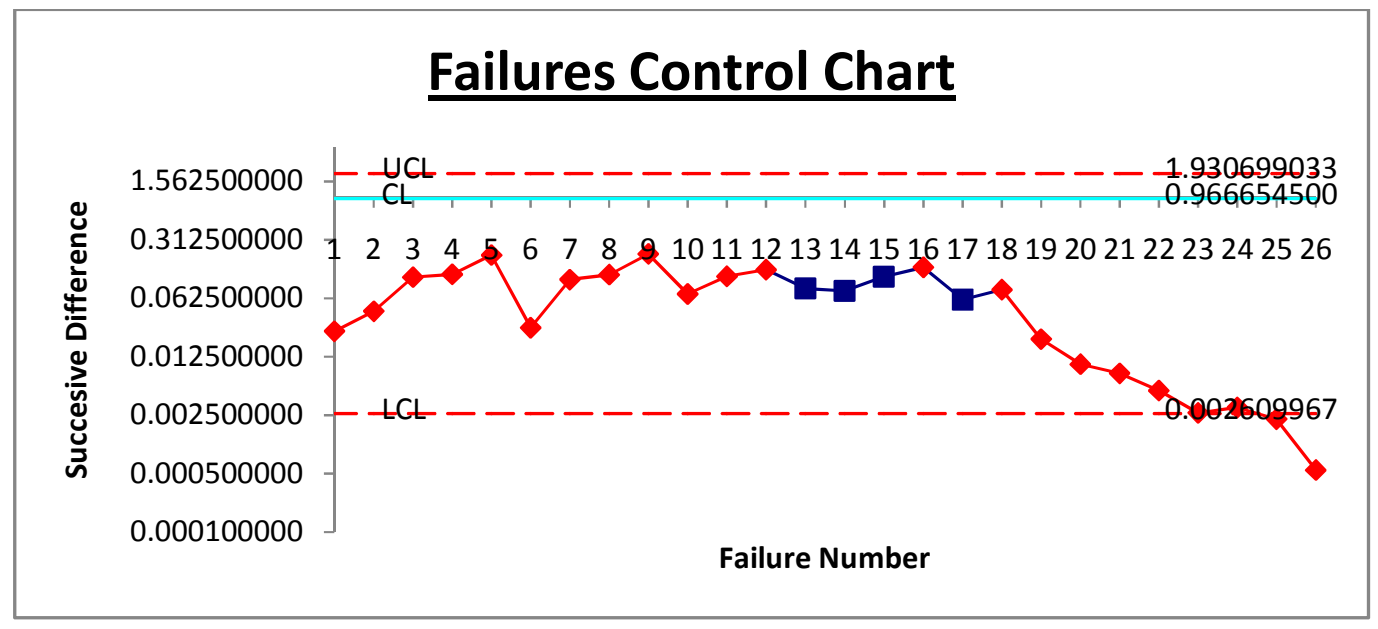

Fig 2 : Failures Control Chart of Table 4

\section{CONCLUSION}

The Failures Control Charts of Fig 1 to 2 have shown out of control signals i.e. below LCL. By observing Failures Control Charts, we identified that failures situation is detected at an early stages. The early detection of software failure will improve the software reliability. When the control signals are below LCL, it is likely that there are assignable causes leading to significant process deterioration and it should be investigated. Hence, we conclude that our control mechanism proposed in this chapter with order statistics approach giving a positive recommendation for its use to estimate whether the process is in control or out of control.

\section{ACKNOWLEDGMENTS}

Our thanks to Department of Computer Science and Engineering; Department of Statistics, Acharya Nagarjuna University; Department of Computer Sceince, Annabathuni Satyanaraya Degree College, Tenali, for providing necessary facilities to carryout the research work.

\section{REFERENCES}

[1] N. Boffoli, G. Bruno, D. Cavivano, G. Mastelloni; Statistical process control for Software: a systematic approach; 2008 ACM 978-1-595933-971-5/08/10.

[2] K. U. Sargut, O. Demirors; Utilization of statistical process control (SPC) in emergent software organizations: Pitfallsand suggestions; Springer Science + Business media Inc. 2006.

[3] Burr,A. and Owen ,M.1996. Statistical Methods for Software quality . Thomson publishing Company. ISBN 1-85032-171-X.

[4] Carleton, A.D. and Florac, A.W. 1999. Statistically controlling the Software process. The 99 SEI Software Engineering Symposimn, Software Engineering Institute, Carnegie Mellon University.

[5] Mutsumi Komuro; Experiences of Applying SPC Techniques to software development processes; 2006 ACM 1-59593-085-x/06/0005.

[6] Arak M. Mathai ;Order Statistics from a Logistic Dstribution and Applications to Survival and Reliability Analysis;IEEE Transactions on Reliability, vol.52, No.2; 2003

[7] Balakrishnan.N., Clifford Cohen; Order Statistics and Inference; Academic Press inc.;1991. 
International Journal of Computer Science, Engineering and Applications (IJCSEA) Vol.1, No.4, August 2011

[8] R.SatyaPrasad, “ Software Reliability with SPC”; International Journal of Computer Science and Emerging Technologies; Vol 2, issue 2, April 2011. 233-237

[9] Ronald P.Anjard;SPC CHART selection process;Pergaman 0026-27(1995)00119-0Elsevier science ltd.

[10] Hong Pharm; System Reliability; Springer;2005;Page No.281

[11] M.Xie, T.N. Goh, P. Rajan; Some effective control chart procedures for reliability monitoring; Elsevier science Ltd, Reliability Engineering and system safety 77(2002) 143- 150

[12] R.satyaprasad, Half Logistic Software Reliability Growth Model,Ph.D. Thesis,2007

Authors

K.Ramchand H Rao, received Master's degree in Technology with Computer Science from Dr. M.G.R University, Chennai, Tamilnadu, India, . He is currently working as Associate Professor and Head of the Department, in the Department of Computer Science, A.S.N. Degree College, Tenali, which is affiliated to Acharya Nagarjuna University. He has 18 years teaching experience and 2 years of Industry experience at Morgan Stanly, USA as Software Analyst. He is currently pursuing Ph.D., at Department of Computer Science and Engineering, Acharya Nagarjuna University, Guntur, Andhra Pradesh, India. His research area is software Engineering. He has published several papers in National \& International Journals.

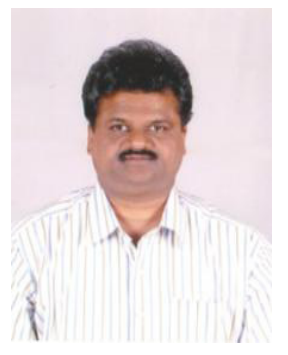

Dr. R. Satya Prasad received Ph.D. degree in Computer Science in the faculty of Engineering in 2007 from Acharya Nagarjuna University, Andhra Pradesh. He received gold medal from Acharya Nagarjuna University for his out standing performance in Masters Degree. He is currently working as Associate Professor and H.O.D, in the Department of Computer Science \& Engineering, Acharya Nagarjuna University. His current research is focused on Software Engineering, Software reliability. He has published several papers in National \& International Journals.

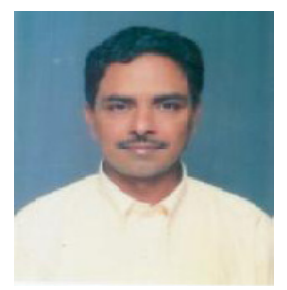

R.R.L.Kantam is professor of statistics at Acharya Nagarjuna University,GunturIndia. He has 31 years of teaching experience in statistics at Under Graduate and Post Graduate programs. As researcher in Statistics, he has successfully guided many students for M.Phil and Ph.D. in statistics. He has authored more than 60 research publications appeared various statistics and computer science journals published in India and other countries like US, UK, Germany, Prakistan, Srilanka and Bangladesh. He has been a referee for Journal of Applied Statistics (U.K), METRON (Italy), Pakistan Journal of Statistics (Pakistan), IAPQR - Transactions (India), Assam Statistical Review (India) and Gujarat Statistical Review (India). He has been a special speaker in technical sessions of a number of Seminars and Conferences. His areas of research interest are Statistical Inference, Reliability Studies, Quality Control Methods and Actuarial Statistics. As a teacher his present teaching areas are Probability Theory, Reliability, and Actuarial Statistics. His earlier teaching topics include Statistical Inference, Mathematical Analysis, Operations Research, Econometrics, Statistical Quality control, Measure theory. 